\title{
Continuous Positive Airway Pressure Treatment and Depression in Adults with Coronary Artery Disease and Nonsleepy Obstructive Sleep Apnea
}

\section{A Secondary Analysis of the RICCADSA Trial}

\author{
Baran Balcan ${ }^{1}$, Erik Thunström ${ }^{2,3}$, Patrick J. Strollo Jr. ${ }^{4}$, and Yüksel Peker ${ }^{3,4,5,6}$ \\ ${ }^{1}$ Department of Pulmonary Medicine, School of Medicine, Marmara University, Istanbul, Turkey; ${ }^{2}$ Department of Cardiology, Sahlgrenska \\ University Hospital/Ostra, Gothenburg, Sweden; ${ }^{3}$ Sahlgrenska Academy, University of Gothenburg, Gothenburg, Sweden; ${ }^{4}$ Division of \\ Pulmonary, Allergy, and Critical Care Medicine, University of Pittsburgh School of Medicine, Pittsburgh, Pennsylvania; ${ }^{5}$ Department of \\ Clinical Sciences, Respiratory Medicine and Allergology, Faculty of Medicine, Lund University, Lund, Sweden; and ${ }^{6}$ Department of \\ Pulmonary Medicine, School of Medicine, Koc University, Istanbul, Turkey
}

ORCID IDs: 0000-0003-1804-1970 (B.B.); 0000-0002-9134-2107 (E.T.); 0000-0002-2065-9641 (P.J.S.); 0000-0001-9067-6538 (Y.P.).

\begin{abstract}
Rationale: Obstructive sleep apnea (OSA) and depression are common among adults with coronary artery disease (CAD).
\end{abstract}

Objectives: To determine the impact of continuous positive airway pressure (CPAP) treatment on depression in adults with $\mathrm{CAD}$ and nonsleepy OSA.

Methods: This was a secondary analysis of the RICCADSA (Randomized Intervention with CPAP in CAD and Sleep Apnea) trial, conducted in Sweden between 2005 and 2013. Adults with CAD and nonsleepy OSA (apnea-hypopnea index $\geqslant 15 / h$, and Epworth Sleepiness Scale $<10$ at baseline) and complete Zung Self-Rating Depression Scale (SDS) questionnaires at baseline, after 3 and 12 months, were included. Participants analyzed in their randomized arm were CPAP $(n=99)$ or no-CPAP $(n=104)$. Depression was defined as a Zung SDS score of 50 or greater. The primary outcome was the between-group difference in the absolute change in the SDS score from baseline.

Results: No significant between-group differences were observed in SDS scores during follow-up in the entire study sample. Among the 56 participants with an SDS of 50 or greater at baseline (27.6\%), the mean $( \pm \mathrm{SD})$ baseline SDS was $55.0( \pm 5.5)$ in the CPAP group, and $53.9( \pm 4.0)$ in the no-CPAP group. In the CPAP group, SDS scores decreased at 3 months $(47.2 \pm 8.2)$ and 12 months $(45.8 \pm 7.6)$, but remained stable in the no-CPAP group at 3 months $(53.1 \pm 8.0)$ and 12 months $(52.6 \pm 8.1)(P=0.01)$. The proportion with depression decreased from $30.3 \%$ at baseline to $16.2 \%$ after 3 months, and to $13.1 \%$ after 12 months in the CPAP group, from $25.0 \%$ at baseline to $23.1 \%$ after 3 months, and to $24.0 \%$ after 12 months in the no-CPAP group $(P=0.001)$. Moreover, there was an association between the duration of CPAP usage $(\mathrm{h} / \mathrm{night})$ and the longitudinal decline in SDS score $(r=0.46 ; P<0.001)$. CPAP usage categories $(3,4$, and $5 \mathrm{~h} /$ night $)$ were significantly associated with improvement in SDS (odds ratio $=3.92,4.45$, and 4.89 , respectively) in multivariate analyses adjusted for age, sex, body mass index, left ventricular ejection fraction, apnea-hypopnea index, and Epworth Sleepiness Scale at baseline.

Conclusions: Among adults with depression, nonsleepy OSA, and CAD, 3 months of CPAP treatment improved depression scores.

The improvement in mood persisted up to 12 months. An on-treatment adjusted analysis confirmed these findings.

Clinical trial registered with www.clinicaltrials.gov (NCT00519597).

Keywords: positive airway pressure; coronary artery disease; depression; sleep apnea

(Received in original form March 12, 2018; accepted in final form August 20, 2018)

Author Contributions: Y.P. designed the study; Y.P. and E.T. performed the patient recruitment and clinical follow-ups; B.B. and Y.P. performed the statistical analysis; Y.P. obtained study funding and takes full responsibility for the work as a whole, including the study design, access to data, and the decision to submit and publish the manuscript; all authors interpreted the data, prepared the manuscript, and drafted and approved the manuscript in its final form.

Correspondence and requests for reprints should be addressed to Yüksel Peker, M.D., Ph.D., Department of Pulmonary Medicine, School of Medicine, Koc University, Topkapi Mah, Davutpasa caddesi, No 4, TR 34010 Zeytinburnu, Istanbul, Turkey. E-mail: yuksel.peker@lungall.gu.se.

This article has an online supplement, which is accessible from this issue's table of contents at www.atsjournals.org.

Ann Am Thorac Soc Vol 16, No 1, pp 62-70, Jan 2019

Copyright (C) 2019 by the American Thoracic Society

DOI: 10.1513/AnnalsATS.201803-1740C

Internet address: www.atsjournals.org 
There is an increasing body of evidence regarding an association between depression and cardiovascular disease (CVD) (1). It was suggested that depression may trigger a heart attack, and depressive episodes may have adverse effects in patients with coronary artery disease (CAD) (2). It was also reported that individuals who had depression in their past medical history are at fourfold higher risk of having a heart attack compared with the controls (3). Moreover, there are data demonstrating an association between the severity of depression and cardiac death (4). Stress reactivity, exacerbated by depression, has been linked to autonomic dysfunction (5), and increased inflammatory activity (such as high c-reactive protein, IL-6), which also may contribute to the development of CVD in those patients (6).

Obstructive sleep apnea (OSA) is characterized by repeated episodes of upper airway collapse during sleep with disturbances in arterial oxygen saturation, and daytime symptoms, such as headache in the morning and excessive daytime sleepiness (EDS) (7). OSA has been linked to increased cardiovascular morbidity and mortality $(8,9)$. There are also data suggesting an increased occurrence of depression in patients with OSA, and this may contribute to EDS in those patients (10). Likewise, OSA has been reported to be more common in patients with depression (11).

Continuous positive airway pressure (CPAP) is the first-choice treatment for patients with OSA with EDS $(12,13)$. Early randomized, controlled trials (RCTs) have demonstrated beneficial effects of CPAP with regard to quality of life in addition to improvement in EDS in sleep clinic cohorts (14-16). Less is known regarding the association between OSA and depression, as well as the effect of CPAP on mood in patients with CAD with comorbid nonsleepy OSA. In the SAVE (Sleep Apnea Cardiovascular Endpoints Study) trial, comprising 2,410 patients with CAD and cerebrovascular disease, CPAP treatment improved mood in the intention-to-treat (ITT) population, though the results were neutral for the primary cardiovascular outcomes (17).
The RICCADSA (Randomized Intervention with CPAP in CAD and OSA) trial was designed to address the impact of CPAP on the composite of repeat revascularization, myocardial infarction, stroke, and cardiovascular mortality in patients with CAD and nonsleepy OSA (18). The current work evaluated the effect of CPAP on depressive mood in the same cohort as one of the secondary outcomes of the RICCADSA trial.

\section{Methods}

\section{Study Design and Participants}

The study sample has been described in detail previously (19). Consecutive patients with CAD who underwent percutaneous coronary intervention or coronary artery by-pass grafting (CABG) within 6 months before recruitment in the Skaraborg County of West Götaland, Sweden, were invited to participate. The patient recruitment was conducted between 2005 and 2010, and the follow-up for the primary outcomes was ended in May 2013 (19). As outlined in Figure 1, the patients with CAD with nonsleepy OSA (apnea-hypopnea index $[\mathrm{AHI}] \geqslant 15 / \mathrm{h}$ ) on the baseline cardiorespiratory polygraphy (CRPG), and Epworth Sleepiness Scale (ESS) less than 10, were randomized to CPAP or no-CPAP in the entire cohort (1:1 assignment, stratified by sex and revascularization type [percutaneous coronary intervention/ $\mathrm{CABG}]$ ). For the current protocol, those with nonsleepy OSA, who completed Zung Self-Rating Depression Scale (SDS) questionnaires at baseline, after 3 and 12 months, were included. The study protocol was approved by the Ethics Committee of the Medical Faculty of the University of Gothenburg (approval no. 207-05; 09.13.2005; amendment T744-10; 11.26.2010; amendment T512-11;

06.16.2011), and written informed consent was provided by all patients. The trial was registered (FoU i Sverige-Research and Development in Sweden; researchweb.org; VGSKAS-4731; 04.29.2005; and www. clinicaltrials.gov; NCT00519597).

\section{CRPG}

The portable CRPG was performed with the Embletta Portable Digital System device (Embla). As described in detail previously (19), the CRPG system included a nasal pressure detector, two respiratory belts for thoraco-abdominal movements and body position, and a finger pulse oximeter for detecting heart rate and oxyhemoglobin saturation (oxygen saturation as measured by pulse oximetry). Apnea was defined as at least $90 \%$ cessation of airflow, and hypopnea was defined as at least a $50 \%$ reduction in thoracoabdominal movement and/or in nasal pressure amplitude for 10 seconds or longer, regardless of desaturations (20). In addition, the total number of significant oxygen saturation as measured by pulse oximetry decreases $(\geqslant 4 \%$ from the immediately preceding baseline) was scored, and the oxygen desaturation index (ODI) was calculated as the number of significant $(\geqslant 4 \%)$ desaturations per hour of estimated sleep. Events with a clear reduction in thoracoabdominal movement and/or in the nasal pressure amplitude for at least 10 seconds were also scored as hypopneas if there was a significant desaturation of at least 4\% (20).

\section{ESS}

Daytime sleepiness was assessed by the ESS questionnaire (21) containing eight questions to address the chance of dozing off under eight different scenarios in the past month. The total score ranges between 0 and 24. A cut-off value of 10 or more for the ESS score was used for categorizing the patients with EDS.

\section{Zung SDS}

All participants were asked to complete Zung SDS at baseline and at follow-ups. The Zung SDS is a widely accepted questionnaire that provides both a total score and a categorical rating of depression (22). In total, 20 items are included, and the rating scale is scored from 1 to 4 points, which gives a raw total score from 20 to 80 . Most answers go in order of 1 (a little of the time) to 4 (most of the time). However, questions $2,5,6,11$, $12,14,16,17,18$, and 20 are scored in the opposite order, as they represent

Supported by Swedish Research Council grants 521-2011-537 and 521-2013-3439, Swedish Heart-Lung Foundation grants 20080592, 20090708, and 20100664), "Agreement Concerning Research and Education of Doctors" of Västra Götalandsregionen grants ALFGBG-11538 and ALFGBG-150801, research fund at Skaraborg Hospital grants VGSKAS-4731, VGSKAS-5908, VGSKAS-9134, VGSKAS-14781, VGSKAS-40271, and VGSKAS-116431, Skaraborg Research and Development Council grant VGFOUSKB-46371, the Heart Foundation of Kärnsjukhuset, the ResMed Foundation, and ResMed Ltd.; ResMed Sweden provided some of the sleep recording devices and technical support. 
1,291 patients with CAD were assessed for eligibility between December 2005 and November 2010

629 were excluded 32 had know OSA 597 were not interested in the study

662 underwent cardio-respiratory polygraphy at home and answered the ESS questionnaire

151 were excluded

101 had borderline OSA (AHI 5.0-14.9 $\mathrm{h}^{-1}$ ) 21 had dominantly CSA/CSR

29 declined further investigations

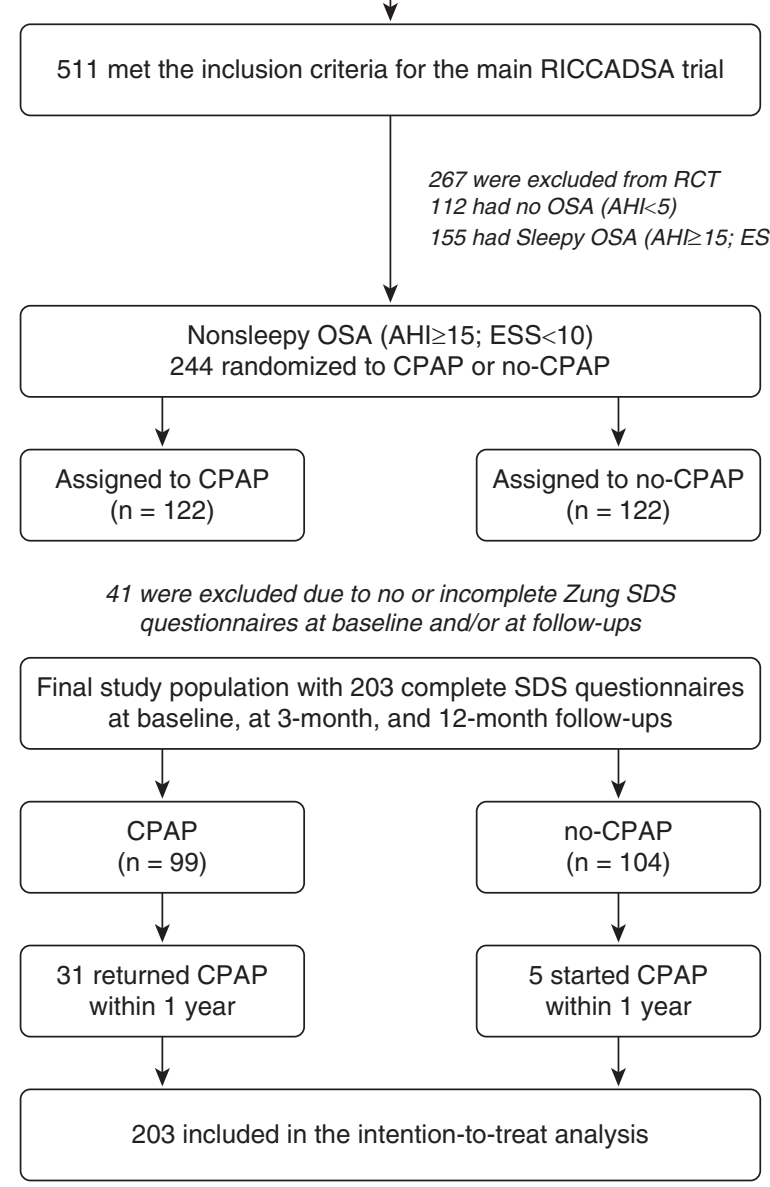

Figure 1. Flow of study participants. $A H I=$ apnea-hypopnea index; $C A D=$ coronary artery disease; $\mathrm{CPAP}=$ continuous positive airway pressure; CSA/CSR = central sleep apnea-Cheyne Stokes respiration; ESS = Epworth Sleepiness Scale; OSA = obstructive sleep apnea; RCT = randomized, controlled trial; RICCADSA = Randomized Intervention with CPAP in CAD and Sleep Apnea; SDS = Self-Rating Depression Scale.

positive/nondepression statements. The raw score is, then, multiplied by 1.25 , resulting in a total range of $25-100$. The subjects with a score below 50 were categorized as normal, the subjects with scores between 50 and 59 as having mild depression, between 60 and 69 as having moderate depression, and the ones with the scores of 70 or greater as having severe depression, respectively (22). The details of the questionnaire, as well as the scoring manual, are provided in online supplement Table E1. As question number 2 ("morning is when I feel the best") as well as question number 4 ("I have trouble sleeping at night") may overlap sleep apnea related symptoms, a separate calculation was performed excluding these items from the total scores. All answers were entered into the database by a research nurse blinded to the group allocation and clinical data.

\section{Comorbidities}

As described previously, baseline anthropometrics, smoking habits, and medical histories of the entire study population were obtained from the medical records (19). Obesity was defined as a body mass index (BMI) of $30 \mathrm{~kg} / \mathrm{m}^{2}$ or greater (23).

\section{Group Assignment, Randomization, Interventions, and Follow-Up}

In the entire RICCADSA cohort, the group assignment was based on the CRPG results and the ESS scores (Figure 1). Patients with nonsleepy OSA randomized to CPAP were provided with an automatic (self-titrating) CPAP device (S8 or S9; ResMed) with a nasal or full-face mask and humidifier. As described previously, all patients receiving CPAP were contacted by telephone after 1 week, and all patients were followed in the clinic after 1 month, 3 months, 6 months, 1 year, and then yearly until the end of the main study (19).

\section{Adherence to CPAP}

All patients allocated to CPAP brought their devices to the clinic at each scheduled follow-up visit, and hours of CPAP use were downloaded from the device and recorded. All technical adjustments were done according to the clinical routines by the sleep medicine unit staff. Patients who returned the devices were followed as part of the treatment arm as defined in the ITT analysis (19).

\section{Outcomes and the Sample Size}

The main outcome of the current study, which was one of the secondary endpoints of the RICCADSA trial, was the betweengroup difference in absolute change in the SDS scores compared with baseline. In addition, a categorical variable, "improvement in mood," was defined for the depression group (SDS score $\geqslant 50$ ) as change to "no depression" (SDS score $<50$ ) at 1 -year follow-up. The sample size estimation for the RICCADSA trial was based on the calculations for the primary endpoints, and no specific 
power estimate was done for the current study.

\section{Statistical Analysis}

Data are shown as mean ( \pm SD or SEM) or 95\% confidence interval for continuous variables, and categorical variables are represented as numbers (percentages). An independently sampled $t$ test was used for between-group differences in means, and the chi-square test (or, when appropriate, Fisher's exact test) was used to compare categorical variables. Repeated measures of SDS scores were analyzed using a two-way ANOVA. Pearson correlation coefficients were used for analyzing the association between change in SDS score from baseline and accumulated CPAP hours per night at 1-year follow-up for the whole group with depression at baseline. Status change from depressed to nondepressed, as well as nondepressed to depressed, was evaluated by $\mathrm{McNemar}$ test. Paired $t$ test was used for changes from baseline within the groups. Logistic regression analysis was used among depressive patients at baseline to determine the variables associated with improvement in mood, and odds ratios (ORs) with 95\% confidence interval were reported. All statistical tests were two sided, and a $P$ value less than 0.05 was considered significant. Statistical analysis was performed using the Statistical Package for Social Sciences, version 22.0 for Windows system (SPSS Inc.).

\section{Results}

\section{Patient Population and Baseline Characteristics}

A total of 244 patients with CAD with nonsleepy OSA was included in the main trial (Figure 1). After excluding 41 patients with no or incomplete SDS questionnaires (at baseline $[n=6]$ and/or at follow-ups $[n=35]$ ), 203 (mean age $=65.79 \pm 8.1 \mathrm{yr}$; $83 \%$ men) remained for the current protocol. In the entire study population, there were no significant differences in the SDS scores at baseline between the CPAP and no-CPAP groups, and 56 patients $(27.6 \%)$ had SDS scores of 50 or greater $(30.3 \%$ in the CPAP group vs. $25.0 \%$ in the no-CPAP group) (Table 1 ). Hypertension and diabetes mellitus tended to be more common among the patients allocated to CPAP, whereas other variables were similar at baseline (Table 1). In the whole group, only seven patients $(3.6 \%)$

Table 1. Baseline characteristics of the study sample with coronary artery disease and nonsleepy obstructive sleep apnea $(n=203)$

\begin{tabular}{|c|c|c|}
\hline Characteristics & $\begin{array}{c}\text { CPAP } \\
(n=99)\end{array}$ & $\begin{array}{r}\text { No CPAP } \\
(n=104)\end{array}$ \\
\hline $\begin{array}{l}\text { Age, yr } \\
\text { Age } \geqslant 65 \mathrm{yr}, \% \\
\text { BMI, } \mathrm{kg} / \mathrm{m}^{2} \\
\text { Obesity, \% } \\
\text { Female sex, \% } \\
\text { AHI, events/h } \\
\text { ODI, events/h } \\
\text { ESS score } \\
\text { CABG before inclusion, \% } \\
\text { Current smoker, \% } \\
\text { AMl at baseline, \% } \\
\text { LVEF } \\
\text { LVEF <50\% at baseline, \% } \\
\text { Former revascularization, \% } \\
\text { Hypertension, \% } \\
\text { Diabetes mellitus, \% } \\
\text { Stroke, \% } \\
\text { Pulmonary disease, \% } \\
\text { Antidepressive medication, \% } \\
\text { Zung SDS score at baseline } \\
\text { Depression (SDS } \geqslant 50 \text { ) at baseline, \% }\end{array}$ & $\begin{array}{c}65.3 \pm 8.1 \\
53.5 \\
28.2 \pm 3.5 \\
27.3 \\
18.2 \\
27.8 \pm 11.9 \\
16.0 \pm 9.9 \\
5.7 \pm 2.3 \\
25.3 \\
15.2 \\
57.6 \\
57.3 \pm 8.5 \\
11.1 \\
19.2 \\
70.7 \\
28.3 \\
7.1 \\
4.0 \\
4.2 \\
42.9 \pm 9.5 \\
30.3\end{array}$ & $\begin{array}{l}66.1 \pm 8.2 \\
53.8 \\
28.5 \pm 3.5 \\
27.9 \\
16.3 \\
29.5 \pm 14.5 \\
16.3 \pm 12.1 \\
5.5 \pm 2.2 \\
26.0 \\
13.5 \\
50.0 \\
56.4 \pm 9.7 \\
17.3 \\
17.3 \\
58.7 \\
18.3 \\
8.7 \\
8.7 \\
2.9 \\
41.9 \pm 8.8 \\
25.0\end{array}$ \\
\hline
\end{tabular}

Definitions of abbreviations: $\mathrm{AHI}=$ apnea-hypopnea index; $\mathrm{AMI}=$ acute myocardial infarction; $\mathrm{BMI}=$ body mass index; $C A B G$ = coronary artery bypass graft; $C P A P=$ continuous positive airway pressure; ESS = Epworth Sleepiness Scale; LVEF = left ventricular ejection fraction; ODI = oxygen desaturation index; SDS = Self-Rating Depression Scale.

were on antidepressive medication. Within the subgroup of patients with depression at baseline, no meaningful differences were observed between the CPAP and no-CPAP arms, except BMI, which was higher in the no-CPAP group (Table 2). When excluding the two items overlapping with sleep, raw total scores decreased from 34.5 ( \pm 7.6$)$ to $30.4( \pm 6.9)$ in the CPAP group, and from 33.6 ( \pm 6.9$)$ to 29.5 ( \pm 6.5$)$ in the no-CPAP group without any meaningful betweengroup difference. In total, $14.1 \%$ in the CPAP group and $20.2 \%$ in the no-CPAP group had answered as "little of the time" to the item "morning is when I feel the best," whereas $5.1 \%$ in the CPAP group and $2.9 \%$ in the no-CPAP group had replied as "most of the time" to the item "I have trouble sleeping at night," respectively. Almost one-fourth of the cohort had answered "a little of the time" to the item "I still enjoy sex," with no between-group differences. The detailed distribution of the items within the groups is provided in online supplement Table E2.

\section{CPAP Adherence}

In the CPAP group, 31 (31.3\%) returned the devices within 12 months. Among 68 participants remaining on CPAP,
$53(77.9 \%)$ were using the device at least $3 \mathrm{~h} /$ night, $44(64.7 \%)$ at least $4 \mathrm{~h} /$ night, and $33(48.5 \%)$ at least $5 \mathrm{~h} /$ night, respectively. For the nonoverlapping categories, 21 (30.1\%) were using the device between 4 and $6 \mathrm{~h} / \mathrm{night}$, and $23(33.8 \%)$ were using it at least $6 \mathrm{~h} /$ night. In the no-CPAP group, five patients $(4.6 \%)$ had crossed over to the CPAP arm within the follow-up period (Figure 1). As shown in online supplement Table E3, participants who returned the device within 12 months were slightly older, but did not differ by sex, BMI, obesity, comorbidities, and baseline AHI, ODI, ESS, and Zung SDS scores compared with those remaining on CPAP. Among participants in the CPAP arm with depression at baseline $(n=30), 16(53.3 \%)$ were using the device at least $3 \mathrm{~h} /$ night, and 15 (50.0\%) at least $4 \mathrm{~h} /$ night at $1-y r$ follow-up.

\section{Outcomes}

ITT population. As illustrated in Figure 2A, the average Zung SDS scores were similar at baseline and at 3-month and 12-month follow-ups $(42.9 \pm 9.5,39.6 \pm 8.7$, and $39.7 \pm 8.2$, respectively) in the group allocated to CPAP, and $41.9( \pm 8.8), 41.2$ $( \pm 10.1)$, and $41.3( \pm 10.0)$, respectively, 
Table 2. Baseline characteristics of the intention-to-treat population with depression (Zung Self-Rating Depression Scale score $\geqslant 50$ ) at baseline

\section{Characteristics}

CPAP

$(n=30)$

$66.9 \pm 8.7$

60.0

Age $\geqslant 65 \mathrm{yr}, \%$

$\mathrm{BMl}, \mathrm{kg} / \mathrm{m}^{2}$

Obesity, \%

Female sex, \%

AHI, events/h

ODI, events/h

ESS score

CABG before inclusion, \%

Current smoker, \%

AMI at baseline, \%

LVEF

LVEF $<50 \%$ at baseline, $\%$

Former revascularization, \%

Hypertension, \%

Diabetes mellitus, \%

Stroke, \%

Pulmonary disease, \%

Antidepressive medication, \%

Zung SDS score at baseline

Definitions of abbreviations: $\mathrm{AHI}=$ apnea-hypopnea index; $\mathrm{AMI}=$ acute myocardial infarction; $\mathrm{BMI}=$ body mass index; $\mathrm{CABG}=$ coronary artery bypass grafting; $\mathrm{CPAP}=$ continuous positive airway pressure; ESS = Epworth Sleepiness Scale; LVEF = left ventricular ejection fraction; ODI = oxygen desaturation index; SDS = Self-Rating Depression Scale.

in the no-CPAP group $(P=0.53)$. In the subgroup with depression at baseline $(n=$ $56)$, there was a significant reduction from $55.0( \pm 5.5)$ to $47.2( \pm 8.2)$ after 3 months, and $45.8( \pm 7.6)$ after 12 months in the CPAP group, whereas the corresponding values were $53.9( \pm 4.0), 53.1( \pm 8.0)$, and $52.6( \pm 8.1)$, respectively, in the no-CPAP group $(P=0.01$; Figure $2 \mathrm{~B})$. As shown in Table 3, within-group differences regarding the changes at 3 months and 12 months from baseline were significant in the CPAP group. The proportion with depression decreased from $30.3 \%$ at baseline to $13.1 \%$ after 12 months in the CPAP group $(P=0.001)$, from $25.0 \%$ at baseline to $24.0 \%$ after 12 months in the no-CPAP group (not significant). Among 147 patients with no depression at baseline, 4 of $69(5.8 \%)$ in the CPAP group and 6 of $78(7.7 \%)$ in the no-CPAP group demonstrated SDS scores of 50 or greater at 1-year follow-up (not significant). In this subgroup with no depression at baseline, there was a reduction from 42.9 ( \pm 9.5$)$ to $39.6( \pm 8.7)$ after 3 months, and $39.7( \pm 8.2)$ after 12 months in the CPAP arm, whereas the corresponding values were $41.9( \pm 8.8), 41.2( \pm 10.1)$, and $41.3( \pm 10.0)$, respectively, in the no-CPAP group $(P=0.53)$. No significant changes were observed regarding the items overlapping with sleep at follow-up. As shown in the online supplement, no other specific items were identified to be more affected by CPAP, suggesting that the changes in the SDS scores were based on the heterogeneous components of the questionnaire. No changes were observed regarding the antidepressive medications during the follow-up period, and none of the participants were hospitalized due to depression.

On-treatment population. In post hoc analysis of the 56 patients with depression at baseline, there was a reduction from $54.7( \pm 1.2)$ to $46.7( \pm 2.0)$ after 3 months, and to $44.7( \pm 1.9)$ after 12 months among 18 patients using the CPAP device $4 \mathrm{~h} /$ night or longer, whereas the corresponding values were $54.4( \pm 0.8), 51.5( \pm 1.4)$, and $51.0( \pm 1.3)$, respectively, among 38 patients with CPAP use under $4 \mathrm{~h} /$ night or no-CPAP allocation $(P=0.04)$. There was an association between the duration of CPAP usage (h/night) and the longitudinal decline in SDS score $(r=0.46 ; P<0.001)$. As shown in Table 4, none of the variables, except CPAP usage, was associated with improvement in mood at 1-year follow-up in the univariate logistic regression analysis. CPAP hours per night as continuous variable $(\mathrm{OR}=1.37)$, as well as CPAP usage cut-off categories $(3,4$,
$5 \mathrm{~h} /$ night), remained as significant predictors of improvement in mood ( $\mathrm{OR}=3.92,4.45$, and 4.89 , respectively) in the multivariate analysis adjusted for age, sex, BMI, left ventricular ejection fraction, and AHI and ESS score at baseline (Table 4). Within the CPAP group $(n=99)$, there was a reduction from $43.2( \pm 10.6)$ to $39.3( \pm 8.6)$ after 3 months, and 39.0 ( \pm 8.6$)$ after 12 months among the participants who were using CPAP at least $4 \mathrm{~h} /$ night $(n=44)$, whereas the corresponding values were 42.7 ( \pm 8.6$), 39.8$ ( \pm 8.9$)$, and $40.2( \pm 7.9)$, respectively, among participants who were using the device less than $4 \mathrm{~h} /$ night or returned the device $(n=55 ; P=0.79)$.

\section{Discussion}

The current study demonstrated that CPAP treatment for 3 months improved mood in patients with CAD with nonsleepy OSA, which was maintained after 1 year, among the participants who had depression at baseline. In on-treatment analysis, CPAP use was independently associated with an improvement in mood after adjustment for confounding factors.

To the best of our knowledge, this is the first RCT evaluating the impact of CPAP on depressive mood in a CAD cohort with nonsleepy OSA. In the SAVE trial, comprising a large number of patients with CAD and cerebrovascular disease, CPAP treatment was associated with an improvement in mood in the ITT population after 2 years, based on the Hospital Anxiety and Depression Scale scores (17). However, patients with mild sleepiness $(\mathrm{ESS}=10-15)$ were also included ( $\sim 20 \%$ of the entire population) in that trial, leaving the confounding impact of the reduction in ESS on improvement in mood unaddressed. Our results are supportive for the results in the SAVE trial with an additional explanation suggesting that this improvement is independent of the changes in the ESS scores.

Other previous studies addressing the relationship between OSA and depression, as well as the effect of CPAP, were mainly conducted in sleep clinic cohorts, and the results have not been conclusive (11). CPAP was found to be beneficial in some (24-28), and no impact was found in some others (29-31). Only a few studies were RCTs $(25,27,28,31)$. Redline and colleagues (28) addressed the impact of CPAP on a composite endpoint, including mood, in individuals with mild-to-moderate OSA 

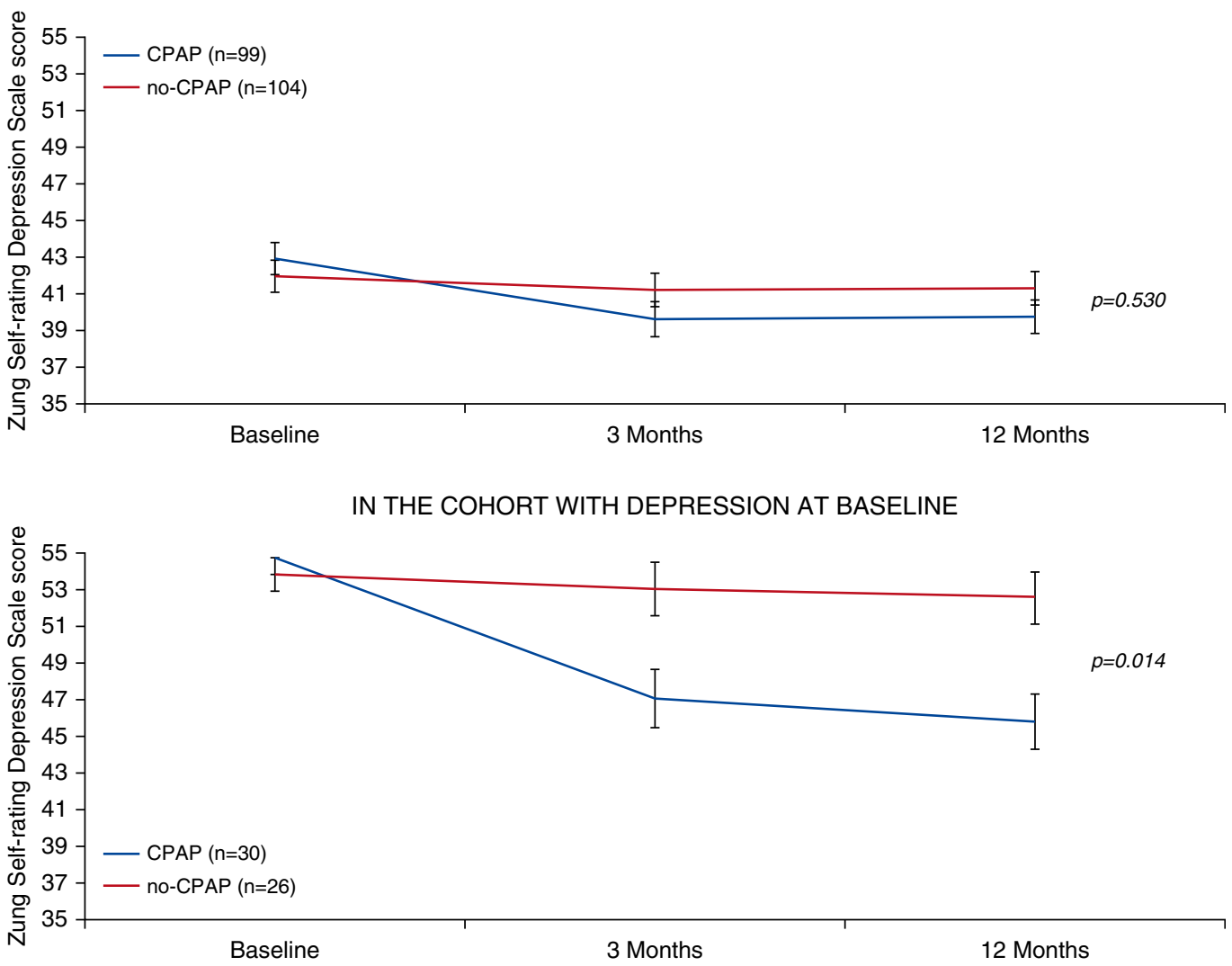

Figure 2. (A) Mean values with standard error of the mean (SEM) at baseline, and after 3 and 12 months, in the continuous positive airway pressure (CPAP) and no-CPAP (control) groups; $P$ values were calculated using analysis of covariance (ANCOVA). (B) Mean values with SEM at baseline, and after 3 and 12 months, among patients with depression at baseline in the CPAP and no-CPAP (control) groups; $P$ values were calculated using ANCOVA. Note: $y$-axes do not start at zero.

(AHI $=5-30$ events/h), but no sleepiness, and reported beneficial effect after 8 weeks of therapy compared with those randomized to conservative treatment. Barnes and colleagues (31) demonstrated in 2004 that

both CPAP and mandibular advancement device were effective in treating EDS of patients with mild-to-moderate OSA compared with placebo tablet, whereas no significant differences were observed in Beck

Table 3. Zung Self-Rating Depression Scale scores at baseline and after 3 and 12 months in the study population with depression at baseline $(n=56)$

\section{CPAP \\ $(n=30)$}

At baseline

After 3 mo

$P$ value $^{\dagger}$

Change from baseline after 3 mo

After 12 mo

$P$ value ${ }^{\dagger}$

Change from baseline after 12 mo

$$
\begin{gathered}
55.0(53.2 \text { to } 56.7) \\
47.1(44.1 \text { to } 50.1) \\
\quad<0.001 \\
-7.8(-10.4 \text { to }-5.3) \\
45.8(42.9 \text { to } 48.6) \\
<0.001
\end{gathered}
$$$$
-9.2(-12.0 \text { to }-6.4)
$$

$P$ Value*

$(n=26)$

$\begin{array}{cc}53.9(52.0 \text { to } 55.9) & 0.44 \\ 53.1(49.9 \text { to } 56.3) & 0.01 \\ 0.48 & \\ -0.9(-3.6 \text { to } 1.9) & 0.001 \\ 52.6(49.6 \text { to } 55.7) & 0.002 \\ 0.24 & \\ -1.3(-4.3 \text { to } 1.7) & <0.001\end{array}$

Definitions of abbreviation: CPAP = continuous positive airway pressure.

Data are presented as mean and 95\% confidence intervals.

${ }^{*}$ For between-group differences (independent sampled $t$ test).

${ }^{\dagger}$ For within-group differences (paired $t$ test).
Depression Scale scores. In another RCT, Lim and colleagues (32) did not find any positive effect of CPAP treatment on depression and daytime symptoms after 2 weeks. In the APPLES (Apnea Positive Pressure Long-term Efficacy Study) study, impact of CPAP on neurocognitive function, as well as on EDS, was evaluated, but there was no report regarding the impact on mood (33). However, the baseline report from a subgroup of the same cohort showed no relationship between mild OSA $(\mathrm{AHI}=$ $5-15 / \mathrm{h}$ ) and worse mood (34). In an early, uncontrolled prospective study, no improvement in Beck Depression Scale scores was observed after 3 and 12 months of CPAP treatment in patients with severe OSA (30). Likewise, in a later study by Gagnadoux and colleagues (29), CPAP therapy did not resolve depressive symptoms in many patients with OSA, and persistent depressive symptoms were strongly associated with EDS. In contrary, beneficial effects of CPAP on mood based on 
Table 4. Variables associated with improvement in mood after 12 month in the study population with Zung Self-Rating Depression Scale score of 50 or greater at baseline $(n=56)$

\begin{tabular}{lccc}
\hline Variables & Odds Ratio & $\mathbf{9 5 \%}$ Cl & P Value \\
& & & \\
Univariate & & & \\
Age, yr & 1.05 & $0.99-1.12$ & 0.12 \\
BMl, kg/m ${ }^{2}$ & 0.97 & $0.83-1.12$ & 0.65 \\
Obesity & 0.63 & $0.21-1.88$ & 0.41 \\
Female sex & 1.00 & $0.29-3.35$ & 1.00 \\
CABG vs. PCl & 0.65 & $0.18-2.37$ & 0.52 \\
Current smoker & 0.50 & $0.13-1.95$ & 0.32 \\
Hypertension & 1.17 & $0.39-3.56$ & 0.78 \\
AMl at baseline & 0.75 & $0.25-2.16$ & 0.59 \\
LVEF at baseline & 1.00 & $0.95-1.06$ & 0.89 \\
Diabetes mellitus & 0.70 & $0.22-2.26$ & 0.56 \\
Stroke & 3.24 & $0.32-33.2$ & 0.32 \\
ESS score at baseline & 0.85 & $0.67-1.07$ & 0.17 \\
Change in ESS score & 0.93 & $0.78-1.13$ & 0.47 \\
AHI & 0.99 & $0.96-1.03$ & 0.68 \\
ODI & 0.99 & $0.95-1.04$ & 0.80 \\
CPAP h/night/all nights & 1.32 & $1.07-1.64$ & 0.01 \\
CPAP usage $\geqslant 3$ h/all nights & 3.18 & $0.99-10.23$ & 0.05 \\
CPAP usage $\geqslant 4$ h/all nights & 3.99 & $1.18-13.50$ & 0.03 \\
CPAP usage $\geqslant 5$ h/all nights & 5.39 & $1.31-22.25$ & 0.02 \\
Multivariate* & & & \\
CPAP h/night/all nights & 1.37 & $1.07-1.75$ & 0.01 \\
CPAP usage $\geqslant 3$ h/night $(n=19)$ & 3.92 & $1.04-14.77$ & 0.04 \\
CPAP usage $\geqslant 4$ h/night $(n=18)$ & 4.45 & $1.15-17.20$ & 0.03 \\
CPAP usage $\geqslant 5$ h/night $(n=14)$ & 4.89 & $1.09-21.96$ & 0.04 \\
\hline
\end{tabular}

Definition of the abbreviations: $\mathrm{AHI}=$ apnea-hypopnea index; $\mathrm{AMI}=$ acute myocardial infarction; $\mathrm{BMI}=$ body mass index; $\mathrm{CABG}=$ coronary artery bypass grafting; $\mathrm{Cl}=$ confidence interval; $\mathrm{CPAP}=$ continuous positive airway pressure; ESS = Epworth Sleepiness Scale; LVEF = left ventricular ejection fraction; $\mathrm{ODI}=$ oxygen desaturation index; $\mathrm{PCl}$ = percutaneous coronary intervention.

*Adjusted for age, female sex, BMI, LVEF, AHI, and ESS score at baseline.

the Zung SDS scores were reported in a smaller, uncontrolled, 2-year follow-up study of 47 patients with OSA (35), as well as in another sleep clinic cohort of 132 patients after 8 weeks of CPAP treatment (9). In a recent multicenter RCT among 307 female patients with moderate-to-severe OSA, Campos-Rodriguez and colleagues (27) demonstrated significant improvement in depression after 3 months of CPAP therapy compared with conservative treatment.

In the current study, we observed depressive mood among $28 \%$ of the revascularized patients with $\mathrm{CAD}$, of whom the vast majority had mildly elevated depressive symptoms. These findings are in line with the study by Thombs and colleagues (36), who reported major depression among 20\% of a hospitalized CAD cohort (36-38). Moreover, Bush and colleagues (39) demonstrated that coexisting depression was associated with an almost fourfold relative risk increase for mortality in patients with CAD adjusted for the other independent predictors (higher age, reduced left ventricular ejection fraction, and diabetes mellitus). In the SAVE trial, no information was available on the percentage of patients with clinically significant depression at baseline, but the ratios were 25-30\% lower in the CPAP group compared with that in the usual-care group at the end of the follow-up (17).

We found no significant change in the SDS scores after 1 year in the entire population. This may be due to several factors. First, CPAP adherence was inadequate in $58 \%$ of randomized patients when applying a cut-off level of $4 \mathrm{~h} /$ night, meaning that average CPAP usage was low in the ITT population. Second, treatments other than CPAP were given as clinically indicated, and were not controlled for in the study protocol. However, the number of patients on antidepressive medication was very low, and none of the participants had started such a medication within the follow-up period. Third, given the existing data suggesting a relationship between EDS and depressive mood (29), it might be possible that the main focus on nonsleepy participants in our study might have contributed to lack of meaningful between-group difference in absolute values in the entire ITT population when the nondepressed participants were considered.

However, when focusing on the subgroup of patients with depressive scores at baseline, CPAP treatment significantly reduced the SDS scores after 3 months, and this improvement was stable even after 12 months in the ITT population. As there was no sham-CPAP treatment in the control arm, this might be a placebo effect, as CPAP allocation in terms of an "additional care" might have influenced the participants' mood in a positive way, with the expectation that CPAP treatment would improve their health. However, the significant relationship between the CPAP hours per night and decrease in SDS scores, independent of the confounding factors in the post hoc analysis, suggests that the impact of CPAP treatment on mood in the participants with depressive symptoms is not only a placebo effect. The usually accepted CPAP adherence in sleep clinic cohorts is defined as $4 \mathrm{~h} /$ night for at least $70 \%$ of the night, which corresponds to an accumulated usage of $2.8 \mathrm{~h} /$ night/all nights (40). Although several cut-off values of CPAP usage were described for improvement in daytime symptoms in sleep clinic cohorts (41), the CPAP usage of at least $4 \mathrm{~h} /$ night was targeted in the latest RCTs for cardiovascular outcomes $(17,18,42)$. Although the post hoc results of this cut-off level were neutral in the SAVE trial for cardiovascular end-points, there was an improvement in depression scores, albeit including the mildly sleepy population (17). This may indicate that improvement in cardiovascular outcomes may require more complete treatment (i.e., CPAP adherence throughout the entire sleep period, and particularly treatment during rapid eye movement sleep), whereas symptomatic benefits can be achieved at the lower levels of CPAP adherence, especially in the symptomatic patients. As the current study was conducted in the patients with nonsleepy OSA at baseline, the significant improvement in mood, independent of changes in sleepiness severity, already at the level of CPAP usage of $3 \mathrm{~h} /$ night is noteworthy.

It is plausible that a depressed patient may be less likely to be adherent to CPAP, as opposed to an optimistic, nondepressed patient. It is also possible that adding an intervention (i.e., CPAP) may improve mood via a placebo effect. Alternatively, being a "CPAP-adherent" patient may contribute to improvement in mood by improving sleep quality and continuity. 


\section{Limitations}

This study has a number of limitations. First, the power estimate for the entire RCT cohort was conducted for the primary outcome (i.e., reduction in composite of repeat revascularization, myocardial infarction, stroke, and cardiovascular mortality), and not for the secondary outcomes assessed in this study. The sample size may not have been large enough to detect significant changes in mood in the entire ITT population, mainly because the CPAP adherence rate was lower than initially expected. However, the positive impact among those with depressive scores at baseline is in line with the results of the SAVE trial, with an additional explanation that this effect is independent of the changes in the ESS scores.

Second, the group allocation was based on the cardiorespiratory sleep studies in comparison to polysomnography, and thus, the total sleep time could not be given exactly. However, the cut-off value for AHI (15/h) chosen for OSA diagnosis was previously shown to be reliable for the Embletta system (43) used in the current trial. The subjects with an AHI of 5.0-14.9 were described as borderline OSA, handled according to the clinical routines, and not included in the RCT arm. Third, the trial was open label, and had no placebo control arm. However, as previously discussed (44), there is no true sham-CPAP or other appropriate placebo for CPAP in a longterm trial in patients with CVD. It is also likely that sham-CPAP consisting of a mask attached to tubing, but without pressure application, would worsen sleep disturbance and mood, and act as a "negative placebo" (45). Fourth, "nonsleepy" OSA in this cohort relied on an ESS threshold, which may not reflect an objective sleepiness. However, this is a largely accepted questionnaire for subjective daytime sleepiness, and other methods, such as Multiple Sleep Latency Test (46), which is recommended as an objective tool, are time consuming and not feasible for the large-scale intervention studies in cardiac populations. Similarly, one may also argue that the Zung SDS questionnaire is a subjective tool, and may not accurately classify depression. However, the proportion of overlapping items with sleep is quite low in this nonsleepy CAD cohort, and the questionnaire is a generally accepted tool to assess mood in clinical cohorts. Finally, results of the on-treatment analysis must be interpreted cautiously, as device usage is patient driven, and self-selection bias cannot be excluded.

\section{Conclusions}

We conclude that CPAP treatment for 3 months reduced SDS scores in patients with CAD with nonsleepy OSA among participants who had depressive mood at baseline. The improvement in mood remained significant at the 12 -month follow-up, and was predicted by CPAP hours per night in an on-treatment analysis adjusted for confounding factors. Thus, adding interventions to standard clinical care of OSA to improve mood may promote CPAP adherence, and, similarly, better adherence to CPAP treatment and alleviation of the underlying OSA may improve mood in patients with CAD.

Author disclosures are available with the text of this article at www.atsjournals.org.

Acknowledgment: The authors gratefully acknowledge sleep technologist Paul Murphy (The Registered Polysomnographic Technologist, European Sleep Technologist) for scoring of the in-hospital polysomnography recordings, research nurse Alen Salkic for data entry of the Zung Self-Rating Depression Scale questionnaires, and research secretary Eija Magnusson for her professional assistance in data entry and coordinating quality control of patient records and data follow-up during the whole trial period.

\section{References}

1 Nicholson A, Kuper H, Hemingway H. Depression as an aetiologic and prognostic factor in coronary heart disease: a meta-analysis of 6362 events among 146538 participants in 54 observational studies. Eur Heart J 2006;27:2763-2774.

2 Lichtman JH, Froelicher ES, Blumenthal JA, Carney RM, Doering LV, Frasure-Smith N, et al.; American Heart Association Statistics Committee of the Council on Epidemiology and Prevention and the Council on Cardiovascular and Stroke Nursing. Depression as a risk factor for poor prognosis among patients with acute coronary syndrome: systematic review and recommendations: a scientific statement from the American Heart Association. Circulation 2014;129:1350-1369.

3 Carney RM, Freedland KE, Miller GE, Jaffe AS. Depression as a risk factor for cardiac mortality and morbidity: a review of potential mechanisms. J Psychosom Res 2002;53:897-902.

4 Lespérance F, Frasure-Smith N, Talajic M, Bourassa MG. Five-year risk of cardiac mortality in relation to initial severity and one-year changes in depression symptoms after myocardial infarction. Circulation 2002; 105:1049-1053.

5 Frisbee JC, Brooks SD, Stanley SC, d'Audiffret AC. An unpredictable chronic mild stress protocol for instigating depressive symptoms, behavioral changes and negative health outcomes in rodents. $J$ Vis Exp 2015;(106):e53109.

6 Moyer CF, Sajuthi D, Tulli H, Williams JK. Synthesis of IL-1 alpha and IL-1 beta by arterial cells in atherosclerosis. Am J Pathol 1991;138: 951-960.

7 Bradley TD, Phillipson EA. Pathogenesis and pathophysiology of the obstructive sleep apnea syndrome. Med Clin North Am 1985;69: 1169-1185.
8 McNamara SG, Grunstein RR, Sullivan CE. Obstructive sleep apnoea. Thorax 1993;48:754-764.

9 Kawahara S, Akashiba T, Akahoshi T, Horie T. Nasal CPAP improves the quality of life and lessens the depressive symptoms in patients with obstructive sleep apnea syndrome. Intern Med 2005;44:422-427.

10 Povitz M, Bolo CE, Heitman SJ, Tsai WH, Wang J, James MT. Effect of treatment of obstructive sleep apnea on depressive symptoms: systematic review and meta-analysis. PLoS Med 2014;11:e1001762.

11 Gupta MA, Simpson FC, Lyons DC. The effect of treating obstructive sleep apnea with positive airway pressure on depression and other subjective symptoms: a systematic review and meta-analysis. Sleep Med Rev 2016;28:55-68.

12 Sullivan CE, Issa FG, Berthon-Jones M, Eves L. Reversal of obstructive sleep apnoea by continuous positive airway pressure applied through the nares. Lancet 1981;1:862-865.

13 Loube DI, Gay PC, Strohl KP, Pack AI, White DP, Collop NA. Indications for positive airway pressure treatment of adult obstructive sleep apnea patients: a consensus statement. Chest 1999;115:863-866.

14 Engleman HM, Martin SE, Kingshott RN, Mackay TW, Deary IJ, Douglas NJ. Randomised placebo controlled trial of daytime function after continuous positive airway pressure (CPAP) therapy for the sleep apnoea/hypopnoea syndrome. Thorax 1998;53:341-345.

15 Jenkinson C, Davies RJ, Mullins R, Stradling JR. Comparison of therapeutic and subtherapeutic nasal continuous positive airway pressure for obstructive sleep apnoea: a randomised prospective parallel trial. Lancet 1999;353:2100-2105.

16 Çelik M, Sarikaya Y, Acar M, Kalenderoğlu A, Doğan S, Kaskalan E, et al. Effect of continuous positive airway pressure treatment on depression, anxiety and perceived stress levels in patients with obstructive sleep apnea syndrome [Article in Turkish]. Turk Psikiyatr Derg 2016;27:244-250. 
17 McEvoy RD, Antic NA, Heeley E, Luo Y, Ou Q, Zhang X, et al.; SAVE Investigators and Coordinators. CPAP for prevention of cardiovascular events in obstructive sleep apnea. N Engl J Med 2016; 375:919-931.

18 Peker Y, Glantz H, Eulenburg C, Wegscheider K, Herlitz J, Thunström E. Effect of positive airway pressure on cardiovascular outcomes in coronary artery disease patients with nonsleepy obstructive sleep apnea: the RICCADSA randomized controlled trial. Am J Respir Crit Care Med 2016;194:613-620.

19 Peker Y, Glantz H, Thunström E, Kallryd A, Herlitz J, Ejdebäck J. Rationale and design of the Randomized Intervention with CPAP in Coronary Artery Disease and Sleep Apnoea-RICCADSA trial. Scand Cardiovasc J 2009;43:24-31.

20 Sleep-related breathing disorders in adults: recommendations for syndrome definition and measurement techniques in clinical research: the report of an American Academy of Sleep Medicine Task Force. Sleep 1999;22:667-689.

21 Johns MW. A new method for measuring daytime sleepiness: the Epworth sleepiness scale. Sleep 1991;14:540-545.

22 Zung WW, Richards CB, Short MJ. Self-rating depression scale in an outpatient clinic: further validation of the SDS. Arch Gen Psychiatry 1965;13:508-515.

23 Obesity: preventing and managing the global epidemic: report of a WHO consultation. World Health Organ Tech Rep Ser 2000;894:i-xii, $1-253$.

24 El-Sherbini AM, Bediwy AS, El-Mitwalli A. Association between obstructive sleep apnea (OSA) and depression and the effect of continuous positive airway pressure (CPAP) treatment. Neuropsychiatr Dis Treat 2011;7:715-721.

25 Haensel A, Norman D, Natarajan L, Bardwell WA, Ancoli-Israel S, Dimsdale JE. Effect of a 2 week CPAP treatment on mood states in patients with obstructive sleep apnea: a double-blind trial. Sleep Breath 2007;11:239-244.

26 Diamanti C, Manali E, Ginieri-Coccossis M, Vougas K, Cholidou K, Markozannes E, et al. Depression, physical activity, energy consumption, and quality of life in OSA patients before and after CPAP treatment. Sleep Breath 2013;17:1159-1168.

27 Campos-Rodriguez F, Queipo-Corona C, Carmona-Bernal C, JuradoGamez B, Cordero-Guevara J, Reyes-Nuñez N, et al.; Spanish Sleep Network. Continuous positive airway pressure improves quality of life in women with obstructive sleep apnea: a randomized controlled trial. Am J Respir Crit Care Med 2016;194:1286-1294.

28 Redline S, Adams N, Strauss ME, Roebuck T, Winters M, Rosenberg C. Improvement of mild sleep-disordered breathing with CPAP compared with conservative therapy. Am J Respir Crit Care Med 1998;157: 858-865.

29 Gagnadoux F, Le Vaillant M, Goupil F, Pigeanne T, Chollet S, Masson P, et al.; IRSR Sleep Cohort Group*. Depressive symptoms before and after long-term CPAP therapy in patients with sleep apnea. Chest 2014;145:1025-1031.

30 Borak J, Cieślicki J, Szelenberger W, Wilczak-Szadkowska H, Koziej M, Zieliński J. Psychopathological characteristics of the consequences of obstructive sleep apnea prior to and tree months after CPAP. Psychiatr Pol 1994;28(3 suppl):33-44.

31 Barnes M, McEvoy RD, Banks S, Tarquinio N, Murray CG, Vowles N, et al. Efficacy of positive airway pressure and oral appliance in mild to moderate obstructive sleep apnea. Am J Respir Crit Care Med 2004; 170:656-664.

32 Lim W, Bardwell WA, Loredo JS, Kim EJ, Ancoli-Israel S, Morgan EE, et al. Neuropsychological effects of 2-week continuous positive airway pressure treatment and supplemental oxygen in patients with obstructive sleep apnea: a randomized placebo-controlled study. $J$ Clin Sleep Med 2007;3:380-386.

33 Kushida CA, Nichols DA, Holmes TH, Quan SF, Walsh JK, Gottlieb DJ, et al. Effects of continuous positive airway pressure on neurocognitive function in obstructive sleep apnea patients: the Apnea Positive Pressure Long-term Efficacy Study (APPLES). Sleep (Basel) 2012;35: 1593-1602.

34 Quan SF, Budhiraja R, Batool-Anwar S, Gottlieb DJ, Eichling P, Patel S, et al. Lack of impact of mild obstructive sleep apnea on sleepiness, mood and quality of life. Southwest J Pulm Crit Care 2014;9:44-56.

35 Yamamoto $\mathrm{H}$, Akashiba T, Kosaka N, Ito D, Horie T. Long-term effects nasal continuous positive airway pressure on daytime sleepiness, mood and traffic accidents in patients with obstructive sleep apnoea. Respir Med 2000;94:87-90.

36 Thombs BD, Bass EB, Ford DE, Stewart KJ, Tsilidis KK, Patel U, et al. Prevalence of depression in survivors of acute myocardial infarction. $J$ Gen Intern Med 2006;21:30-38.

37 Lett HS, Blumenthal JA, Babyak MA, Sherwood A, Strauman T, Robins C, et al. Depression as a risk factor for coronary artery disease: evidence, mechanisms, and treatment. Psychosom Med 2004;66:305-315.

38 Frasure-Smith N, Lespérance F. Reflections on depression as a cardiac risk factor. Psychosom Med 2005;67:S19-S25.

39 Bush DE, Ziegelstein RC, Tayback M, Richter D, Stevens S, Zahalsky H et al. Even minimal symptoms of depression increase mortality risk after acute myocardial infarction. Am J Cardiol 2001;88:337-341.

40 Sawyer AM, Gooneratne NS, Marcus CL, Ofer D, Richards KC, Weaver TE. A systematic review of CPAP adherence across age groups: clinical and empiric insights for developing CPAP adherence interventions. Sleep Med Rev 2011;15:343-356.

41 Weaver TE, Maislin G, Dinges DF, Bloxham T, George CF, Greenberg H, et al. Relationship between hours of CPAP use and achieving normal levels of sleepiness and daily functioning. Sleep 2007;30:711-719.

42 Barbé F, Durán-Cantolla J, Sánchez-de-la-Torre M, Martínez-Alonso M, Carmona C, Barceló A, et al.; Spanish Sleep And Breathing Network. Effect of continuous positive airway pressure on the incidence of hypertension and cardiovascular events in nonsleepy patients with obstructive sleep apnea: a randomized controlled trial. JAMA 2012; 307:2161-2168.

43 Dingli K, Coleman EL, Vennelle M, Finch SP, Wraith PK, Mackay TW, et al. Evaluation of a portable device for diagnosing the sleep apnoea/ hypopnoea syndrome. Eur Respir J 2003;21:253-259.

44 Bradley TD, Logan AG, Floras JS; CANPAP Investigators. Rationale and design of the Canadian Continuous Positive Airway Pressure Trial for Congestive Heart Failure patients with Central Sleep ApneaCANPAP. Can J Cardiol 2001;17:677-684.

45 Leung RS, Tkacova R, Bradley TD. Obstructive sleep apnoea. Lancet 1999;354:1212-1213.

46 Wise MS. Objective measures of sleepiness and wakefulness: application to the real world? J Clin Neurophysiol 2006;23:39-49. 